\title{
Head and Body/Tail Pancreatic Carcinomas Are Not the Same Tumors
}

\author{
David Jérémie Birnbaum ${ }^{1,2,3, *}$, François Bertucci ${ }^{1,2,4}$, Pascal Finetti ${ }^{1}$, Daniel Birnbaum ${ }^{1}$ and \\ Emilie Mamessier ${ }^{1}$ \\ 1 Laboratoire Oncologie Prédictive, Centre de Recherche en Cancérologie de Marseille, INSERM UMR1068, \\ CNRS UMR725, Aix-Marseille Université, 13273 Marseille, France; bertuccif@ipc.unicancer.fr (F.B.); \\ finettip@ipc.unicancer.fr (P.F.); daniel.birnbaum@inserm.fr (D.B.); emilie.mamessier@inserm.fr (E.M.) \\ Faculté de Médecine, Aix-Marseille Université, 13385 Marseille, France \\ Département de Chirurgie Générale et Viscérale, AP-HM, 13015 Marseille, France \\ 4 Département d'Oncologie Médicale, Institut Paoli-Calmettes, 13009 Marseille, France \\ * Correspondence: david.birnbaum10@gmail.com; Tel.: +33-4-91-22-34-77
}

Received: 7 February 2019; Accepted: 2 April 2019; Published: 8 April 2019

Abstract: The association between pancreatic ductal adenocarcinoma (PDAC) location (head vs. Body/Tail (B/T)) and clinical outcome remains controversial. We collected clinicopathological and gene expression data from 249 resected PDAC samples from public data sets, and we compared data between 208 head and $41 \mathrm{~B} / \mathrm{T}$ samples. The 2-year overall survival (OS) was better for the head than for the $\mathrm{B} / \mathrm{T}$ PDACs (44 vs. $27 \%, p=0.043$ ), especially when comparing tumors with similar TNM classification (T3/4N0M0: $67 \%$ vs. $17 \%, p=0.002$ ) or from the same molecular class (squamous subtype: $31 \%$ vs. $0 \%, p<0.0001$ ). Bailey's molecular subtypes were differentially distributed within the two groups, with the immunogenic subtype being underrepresented in the " $\mathrm{B} / \mathrm{T}$ " group $(p=0.005)$. Uni- and multivariate analyses indicated that PDAC anatomic location was an independent prognostic factor. Finally, the supervised analysis identified 334 genes differentially expressed. Genes upregulated in the "head" group suggested lymphocyte activation and pancreas exocrine functions. Genes upregulated in the " $\mathrm{B} / \mathrm{T}$ " group were related to keratinocyte differentiation, in line with the enrichment for squamous phenotype. We identified a robust gene expression signature (GES) associated with B/T PDAC location, suggesting that head and B/T PDAC are different. This GES could serve as an indicator for differential therapeutic management based on PDAC location.

Keywords: pancreatic cancer; tumor location; expression profiling; prognosis; survival

\section{Introduction}

Pancreatic ductal adenocarcinoma (PDAC) is a major public health problem worldwide with 260,000 deaths annually [1], and its incidence is rising [2]. PDAC has the highest mortality rate of all human cancers [3]. Complete surgical removal of the tumor, followed by adjuvant chemotherapy, is the only curative treatment. However, less than $20 \%$ of patients are eligible for surgery [4]. The inoperability and the poor prognosis are due to late diagnosis rapid tumor progression $(>50 \%$ of patients display metastases at diagnosis) [5], early recurrences after resection [6,7], and resistance to systemic therapies. Despite considerable research efforts during the past 20 years, conventional treatment approaches, including surgery, radiation, chemotherapy, and a combination of these, have achieved limited impact. Even after such treatments, most of the patients relapse and succumb from their disease. Molecular studies revealed that this poor benefit might be explained, at least in part, by the high heterogeneity found in pancreatic tumors.

Many studies have demonstrated that cancers of the right and left colons have different molecular characteristics [8-14], suggesting that carcinogenesis in a tissue may differ with tumor location. The 
clinical and biological symptoms are also often related to the location of the tumor. Because the pancreas is also subdivided in multiple anatomic regions-the uncinate process, the head, body, and tail-a long-lasting debate has been initiated to know whether PDAC location could have an impact on the developing tumor $[15,16]$. The fact that cell composition (Langerhans islets), fatty tissue infiltration, innervation, blood supply, and the frequency of the different types of pancreatic tumor (branch duct intrapapillary mucinous carcinoma tumor, mucinous cystic neoplasms) are slightly different between the head and the body/tail (B/T) zones of the pancreas legitimates the question. Studies focusing on the association between PDAC location (head vs. $\mathrm{B} / \mathrm{T}$ ) and clinical outcome (overall survival and disease-free survival) are extremely controversial [17-19]. Some of them reported that, at diagnosis, according to tumor stage, the survival of PDAC located in the $\mathrm{B} / \mathrm{T}$ is better than the postoperative survival of PDAC located in the head [20,21]. In contrast, other studies showed that survival is similar between B/T and head tumor locations [16,22-26]. Finally, the SEER data (Surveillance, Epidemiology and End Results Program by the National Cancer Institute) [27], which has gathered the highest number of PDACs to date (1973-2002), showed that patients with a tumor located in the B/T have a worse survival than those with tumor located in the head (3-year survival: $3.9 \%$ vs. $6.2 \%$ ) [20], and have a higher proportion of distant stage diseases (72.7\% in B/T PDAC vs. $39.2 \%$ in head PDAC) [20]. This was recently confirmed in the Australian Pancreatic Cancer Genome Initiative (APGI) cohort (OS in B/T: 12.1 months versus Head: 22.0 months) [28].

Through in-depth molecular characterization, large-scale genomics provides the opportunity to address such a "long-lasting question". Here, to determine whether head and B/T PDACs are similar or different diseases, we have studied clinicopathological and gene expression data of 249 resected pancreatic carcinoma samples, including 208 head and $41 \mathrm{~B} / \mathrm{T}$ tumors.

\section{Materials and Methods}

\subsection{Gene Expression Data Sets}

We collected clinicopathological and gene expression data of 264 clinical pancreatic carcinoma samples from four public data sets, including 249 operated primary cancer samples with informed cancer localization (Supplementary Table S1).

\subsection{Preanalytic Gene Expression Data Processing}

Before analysis, gene expression data were processed. First, each data set was normalized separately: quantile normalization for the available processed data from Illumina set and Robust Multichip Average (RMA) [29] with the nonparametric quantile algorithm as normalization parameter for the raw Affymetrix data. Normalization was done in $R$ using Bioconductor and associated packages. Second, we mapped hybridization probes across the technological microarray platforms represented in these data sets. We used NetAffx Annotation files to update the Affymetrix gene chips annotations, and both SOURCE and EntrezGene (Homo sapiens gene information db, release from 9 December 2008) to retrieve and update the non-Affymetrix gene chips annotations. Then, all probes were mapped based on their EntrezGeneID. In the case of multiple probes mapped to the same EntrezGeneID, the one with the highest variance in a particular dataset was selected to represent the EntrezGeneID. For the TCGA and Bailey's data, we used the available normalized RNA-Seq data that we log2-transformed. Samples of the four sets were pooled for unsupervised analysis by using COMBAT (empirical Bayes) as batch effect removal method, included in the inSilicoMerging R/Bioconductor package. The merged set included 14,531 genes in log2-transformed data. The accuracy of normalization was verified by principal component analysis (PCA) (Supplementary Figure S1). Hierarchical clustering was done using Cluster program and displayed using Treeview [30]. 


\subsection{Gene Expression Data Analysis}

We first defined the molecular subtypes of pancreatic cancer samples in each data set separately as defined in the original publications: the three Collisson's subtypes [31] were classical, quasi-mesenchymal, and exocrine-like; the two Moffitt's epithelial subtypes [32] were basal-like and classical; the two Moffitt's stromal subtypes were normal and activated; and the four Bailey's subtypes [33] were squamous, pancreatic progenitor, immunogenic, and ADEX. Then, we applied the 20 Gatza's activation pathway signature [34] and the 64 immune and stroma cell types classifiers using the xCell web tool [35]. Finally, to identify a gene expression signature able to distinguish PDAC of the head from PDAC of the body/tail of the pancreas, we applied a supervised analysis using learning and validation sets. The learning set included 137 samples of the TCGA data set, including samples from 116 patients in the "head group" and samples from 21 patients in the "B/T group". To identify the differentially-expressed genes, a moderated $t$-test analysis was applied to expression levels of each gene using linear models with empirical Bayes statistic included in the limma R package [36]. Genes were considered as significantly differentially expressed if they showed an absolute fold change $\geq 2$, a $p$-value $\leq 5 \%$, and a $q$-value $\leq 25 \%$. The classification model was then computed using a metagene-based approach with the significant genes and statistics for ponderation. Using a cut-off of 0 , samples were classified as "head-like" or "body/tail-like". Robustness of the resulting classification model was assessed in the independent validation set $(n=112$ samples, including 92 head samples, and $20 \mathrm{~B} / \mathrm{T}$ samples), i.e., the three remaining sets Analyses were done in $\mathrm{R}$ and associated packages. Ontology analyses were performed using DAVID Bioinformatics Resources 6.8 [37].

\subsection{Statistical Analysis}

Overall survival (OS) was calculated from the date of diagnosis to the date of death from pancreatic cancer. Follow-up was measured from the date of diagnosis to the date of last news for living patients. Survivals were calculated using the Kaplan-Meier method and were compared with the log-rank test. Uni- and multivariate prognostic analyses were done using Cox regression analysis (Wald test). All statistical tests were two-sided at the 5\% level of significance. We followed the reporting REcommendations for tumor MARKer prognostic studies (REMARK criteria) [38].

\section{Results}

\subsection{Patients' Characteristics}

A total of 249 PDAC samples were included in the analysis. Their clinicopathological and molecular data are summarized in Table 1. Briefly, the median age was 66 years (range 32-90) and 117 patients (47\%) were female. Most tumors were of ductal type $(n=219,88 \%)$ and were classified as AJCC stage II tumors $(n=209,84 \%)$. Following the TNM classification, most samples were pT3 tumors $(n=148 ; 79 \%)$, most had at least one lymph node involved ( $n=162 ; 67 \%$ ), but only 10 had detectable metastases at diagnosis $(4 \%)$. Within the whole population, 26 tumors $(10 \%)$ were located in the tail, $15(6 \%)$ in the body, and $208(84 \%)$ in the head of the pancreas. The 2-year OS of the whole population was $41 \%$ (95\% CI: 0.33-0.49), with a median follow-up of 11 months (range: 0-156.4).

Samples were then characterized at the molecular level. The Bailey's classification identified the four subtypes: ADEX (21\%), immunogenic (18\%), pancreatic progenitor (22\%), and squamous (39\%). Regarding the Colisson's classification, 47\%, 35\%, and 18\% of PDACs were classified as classical, exocrine-like, and quasi-mesenchymal, respectively. According to the Moffitt's "Tumor" classification, $45 \%$ of samples were basal-like and $5 \%$ were classical. The Moffitt's "Stroma" classification was distributed as activated (63\%) and normal (37\%). 
Table 1. Clinicopathological and molecular characteristics of patients in the whole population and in each location group.

\begin{tabular}{|c|c|c|c|c|c|c|}
\hline \multirow{2}{*}{ Characteristics } & \multirow{2}{*}{$\mathbf{N}$} & \multirow{2}{*}{ All } & \multicolumn{2}{|c|}{ Pancreas Cancer Site } & \multirow{2}{*}{$p$-Value } & \multirow{2}{*}{ Statistic } \\
\hline & & & Body/Tail & Head & & \\
\hline Age at diagnosis, years & & & & & 0.198 & 0.56 \\
\hline$\leq 60$ & 79 & $79(32 \%)$ & $9(22 \%)$ & $70(34 \%)$ & & $(0.22-1.27)$ \\
\hline$>60$ & 170 & $170(68 \%)$ & $32(78 \%)$ & $138(66 \%)$ & & \\
\hline Sex & & & & & 0.733 & 0.86 \\
\hline Female & 117 & $117(47 \%)$ & $18(44 \%)$ & $99(48 \%)$ & & $(0.41-1.78)$ \\
\hline Male & 132 & $132(53 \%)$ & $23(56 \%)$ & $109(52 \%)$ & & \\
\hline $\begin{array}{l}\text { Pathological tumor size } \\
(\mathrm{pT})\end{array}$ & & & & & 0.049 & \\
\hline pT1 & 10 & $10(5 \%)$ & $5(16 \%)$ & $5(3 \%)$ & & \\
\hline pT2 & 24 & $24(13 \%)$ & $4(13 \%)$ & $20(13 \%)$ & & \\
\hline pT3 & 148 & $148(79 \%)$ & $22(71 \%)$ & $126(81 \%)$ & & \\
\hline pT4 & 5 & $5(3 \%)$ & $0(0 \%)$ & $5(3 \%)$ & & \\
\hline $\begin{array}{c}\text { Pathological lymph node } \\
\text { status }(\mathrm{pN})\end{array}$ & & & & & 0.001 & 3.3 \\
\hline Negative & 79 & $79(33 \%)$ & $22(56 \%)$ & $57(28 \%)$ & & $(1.53-7.1)$ \\
\hline Positive & 162 & $162(67 \%)$ & $17(44 \%)$ & $145(72 \%)$ & & \\
\hline Metastases & & & & & 0.063 & 0.28 \\
\hline Negative & 239 & $239(96 \%)$ & $37(90 \%)$ & $202(97 \%)$ & & $(0.06-1.4)$ \\
\hline Positive & 10 & $10(4 \%)$ & $4(10 \%)$ & $6(3 \%)$ & & \\
\hline AJCC stage & & & & & 0.048 & \\
\hline 1 & 25 & $25(10 \%)$ & $7(17 \%)$ & $18(9 \%)$ & & \\
\hline 2 & 209 & $209(84 \%)$ & $30(73 \%)$ & $179(86 \%)$ & & \\
\hline 3 & 5 & $5(2 \%)$ & $0(0 \%)$ & $5(2 \%)$ & & \\
\hline 4 & 10 & $10(4 \%)$ & $4(10 \%)$ & $6(3 \%)$ & & \\
\hline Pathological grade & & & & & 0.808 & \\
\hline 1 & 21 & $21(9 \%)$ & $2(5 \%)$ & $19(9 \%)$ & & \\
\hline 2 & 137 & 137 (57\%) & $23(57 \%)$ & $114(57 \%)$ & & \\
\hline 3 & 81 & $81(34 \%)$ & $15(38 \%)$ & $66(33 \%)$ & & \\
\hline 4 & 2 & $2(1 \%)$ & $0(0 \%)$ & $2(1 \%)$ & & \\
\hline Bailey subtypes & & & & & 0.006 & \\
\hline ADEX & 52 & $52(21 \%)$ & $7(17 \%)$ & $45(22 \%)$ & & \\
\hline Immunogenic & 45 & $45(18 \%)$ & $1(2 \%)$ & $44(21 \%)$ & & \\
\hline Pancreatic progenitor & 56 & $56(22 \%)$ & $14(34 \%)$ & $42(20 \%)$ & & \\
\hline Squamous & 96 & $96(39 \%)$ & $19(46 \%)$ & $77(37 \%)$ & & \\
\hline Collisson subtypes & & & & & 0.065 & \\
\hline Classical & 118 & $118(47 \%)$ & $24(59 \%)$ & $94(45 \%)$ & & \\
\hline Exocrine-like & 87 & $87(35 \%)$ & $8(20 \%)$ & 79 (38\%) & & \\
\hline Quasi-mesenchymal & 44 & $44(18 \%)$ & $9(22 \%)$ & $35(17 \%)$ & & \\
\hline $\begin{array}{l}\text { Moffitt subtypes, } \\
\text { "Tumor" }\end{array}$ & & & & & 0.395 & 1.4 \\
\hline Basal-like & 112 & $112(45 \%)$ & $21(51 \%)$ & $91(44 \%)$ & & $(0.65-2.8)$ \\
\hline Classical & 137 & $137(55 \%)$ & $20(49 \%)$ & $117(56 \%)$ & & \\
\hline $\begin{array}{l}\text { Moffitt subtypes, } \\
\text { "Stroma" }\end{array}$ & & & & & 0.007 & 3.1 \\
\hline Activated & 154 & $154(63 \%)$ & $32(82 \%)$ & $122(60 \%)$ & & $(1.26-8.72)$ \\
\hline Normal & 90 & $90(37 \%)$ & $7(18 \%)$ & $83(40 \%)$ & & \\
\hline $\begin{array}{l}\text { Follow-up median, } \\
\text { months (min-max) }\end{array}$ & 245 & $11.17(0-156.4)$ & $9.63(0.03-60.25)$ & $10.97(0-156.4)$ & & \\
\hline 2-Year OS (95\% CI) & 245 & $41 \%(0.33-0.49)$ & $27 \%(0.14-0.49)$ & $44 \%(0.36-0.54)$ & 0.044 & \\
\hline
\end{tabular}

ADEX aberrantly differentiated endocrine exocrine; AJCC American Joint Committee on Cancer; CI confidence interval; OS overall survival.

\subsection{Clinicopathological and Molecular Characteristics According to Anatomic Location}

Patients were divided in two groups based on the tumor anatomic location: "head" or "B/ $\mathrm{T}$ " of the pancreas. A total of 208 patients $(84 \%)$ had a "head" PDAC and $41(16 \%)$ had a "B/T" PDAC. As shown in Table 1, patients from the "B/T group" were older $(p=0.039)$ than patients from the "head group", had more pT1 tumors $(p=0.049)$, less pathological lymph node involvement $(p=0.001)$, less tumors from ductal type $(p=0.015)$, and less stage 2 tumors $(p=0.04)$. No difference between the two groups was observed concerning the tumor grade. 
In molecular term, all Bailey's molecular subtypes were represented in both "head" and "B/T" tumors. However, the distribution was significantly different, notably the immunogenic subtype was almost absent from the "B/T group" ( $p=0.0058)$. Accordingly, the immunogenic subtype was more frequent in the "head group" than in the "B/T group" $(p=0.0058)$. No significant difference was observed between the two location groups regarding the Collison's subtypes and the Moffit's tumor subtypes. By contrast, there was more Moffit's activated stroma subtype in the "B/T group" than in the "head group" $(p=0.006)$.

\subsection{OS According to Anatomic Location and Prognostic Analysis}

Median OS was 21.9 months (range: 1-156) in the "head group" and 14.1 months (range: 1-60) in the "B/T group", and the respective 2-year OS were $44 \%$ (95\% CI: $0.36-0.54)$ with a median follow-up of 11 months, versus $27 \%$ (95\% CI: 0.14-0.49) with a median follow-up of 9.6 months, revealing longer survival in the "head group" than in the "B/T group" ( $p=0.0439$; Figure $1 \mathrm{~A})$.

A.

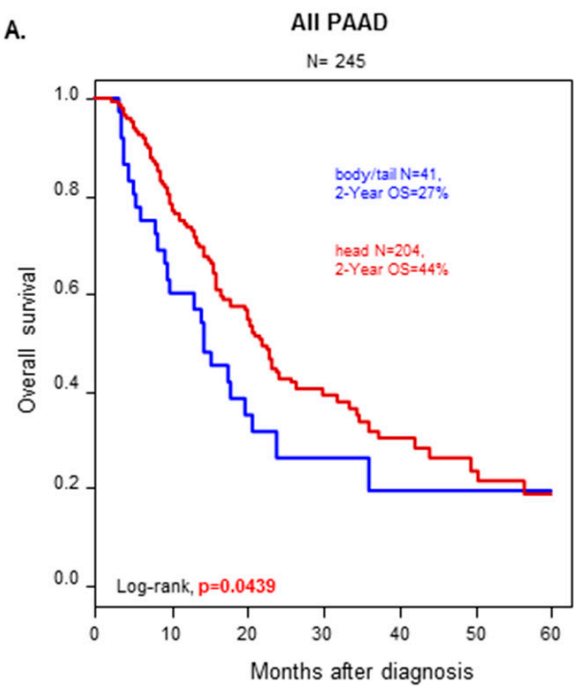

B. T1-2/N0/M0
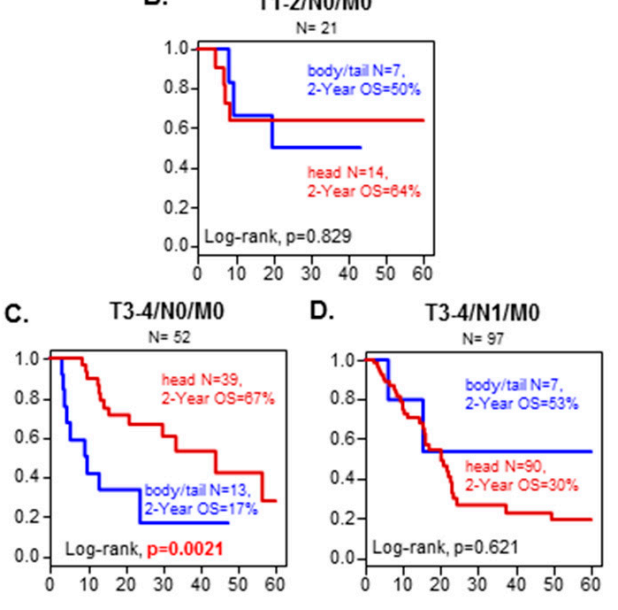

Figure 1. Overall survival in Head or Body/Tail tumors. Kaplan-Meier overall survival (OS) curves according to PDAC location (A). All pancreatic ductal adenocarcinoma (PDAC) and (B) T1/2 N0 M0 tumors. (C) T3/4N0M0 tumors. (D) T3/4 N1 M0 tumors. T1/2N1M0 tumors have less than 10 samples in total and were not graphed). Head samples are in yellow. Body/Tail samples are in blue. The $p$-values (log-rank test) for the comparison between the two classes within each molecular subtype are indicated.

We then compared the prognostic value of the head versus $\mathrm{B} / \mathrm{T}$ anatomic location with that of other clinicopathological variables and molecular subtype classifiers (Bailey, Collisson, and Moffitt). In univariate analysis (Table 2$)$, the $\mathrm{pN}$ status $(p=0.0078)$, the PDAC location $(p=0.0453)$, the Bailey ( $p=0.006$ ), and Moffitt ("Tumor" $p=0.00076$ and "Stroma" $p=0.0002$ ) classifiers were associated with OS (Wald test). The hazard ratio (HR) for death was 0.63 (95\% CI: $0.41-0.99$ ) in the head vs. B/T classes. In multivariate analysis, the PDAC location $(p=0.0047)$, the $\mathrm{pN}$ status $(p=0.0028)$, Moffitt tumor $(p=0.0497)$, and stroma classifiers $(p=0.0232)$ remained significant, suggesting independent prognostic value.

Since AJCC TNM staging is the major prognostic factor used in clinical practice, we analyzed OS in comparable situations, i.e., in groups matched according to the TNM staging. The "head group" conserved a better 2-year OS (67\%) than the "B/T group" $(17 \%)$ when looking at tumors classified as T3-4N0M0 ( $p=0.002$; Figure 1B-D). 
Table 2. Uni- and multivariate Cox regression analyses for overall survival (validation set).

\begin{tabular}{|c|c|c|c|c|c|c|c|}
\hline \multirow{2}{*}{ Characteristics } & & \multicolumn{3}{|c|}{ Univariate } & \multicolumn{3}{|c|}{ Multivariate } \\
\hline & & $\mathbf{N}$ & HR (95\%CI) & $p$-Value & $\mathbf{N}$ & HR (95\%CI) & $p$-Value \\
\hline Age at diagnosis & $>60$ vs. $\leq 60$ & 245 & $0.93(0.63-1.37)$ & 0.708 & & & \\
\hline Sex & male vs. female & 245 & $1.13(0.78-1.62)$ & 0.516 & & & \\
\hline Pathological type & other vs. ductal & 245 & $1.12(0.62-1.99)$ & 0.711 & & & \\
\hline \multirow{3}{*}{ Pathological tumor size (pT) } & 2 vs. 1 & 183 & $1.78(0.37-8.66)$ & 0.290 & & & \\
\hline & 3 vs. 1 & & $2.49(0.61-10.24)$ & & & & \\
\hline & 4 vs. 1 & & $5.88(0.81-42.62)$ & & & & \\
\hline $\begin{array}{l}\text { Pathological lymph node } \\
\text { status (pN) }\end{array}$ & 1 vs. 0 & 237 & $1.77(1.16-2.7)$ & 0.008 & 230 & $2.04(1.28-3.27)$ & 0.003 \\
\hline \multirow[t]{2}{*}{ Metastases } & 1 vs. 0 & 245 & $1.71(0.74-3.92)$ & 0.207 & & & \\
\hline & 2 vs. 1 & 245 & $2.14(0.99-4.63)$ & 0.083 & & & \\
\hline \multirow[t]{3}{*}{ AJCC stage } & 3 vs. 1 & & $4.98(1.01-24.50)$ & & & & \\
\hline & 4 vs. 1 & & $3.50(1.16-10.61)$ & & & & \\
\hline & 2 vs. 1 & 241 & $1.47(0.59-3.69)$ & 0.056 & 230 & $1.38(0.52-3.61)$ & 0.516 \\
\hline \multirow[t]{2}{*}{ Pathological grade } & 3 vs. 1 & & $2.33(0.92-5.89)$ & & 230 & $1.86(0.71-4.89)$ & 0.209 \\
\hline & 4 vs. 1 & & $2.52(0.49-13.13)$ & & 230 & $3.61(0.66-19.82)$ & 0.140 \\
\hline Pancreatic tumor site & head vs. body/tail & 245 & $0.63(0.41-0.99)$ & 0.045 & 230 & $0.46(0.27-0.79)$ & 0.005 \\
\hline \multirow[t]{2}{*}{ KRAS mutation } & MT vs. WT & 137 & $1.2(0.68-2.13)$ & 0.534 & & & \\
\hline & G12D vs. WT & 137 & $1.53(0.79-2.98)$ & 0.317 & & & \\
\hline \multirow[t]{3}{*}{ KRAS mutation, exon 12} & G12R vs. WT & & $1.03(0.39-2.72)$ & & & & \\
\hline & G12V vs. WT & & $0.63(0.26-1.57)$ & & & & \\
\hline & Immunogenic vs. ADEX & 245 & $0.92(0.50-1.70)$ & 0.006 & 230 & $1.64(0.75-3.6)$ & 0.216 \\
\hline \multirow[t]{2}{*}{ Bailey subtypes } & Pancreatic progenitor vs. ADEX & & $0.97(0.54-1.73)$ & & 230 & $1.43(0.68-3)$ & 0.345 \\
\hline & Squamous vs. ADEX & & $1.87(1.13-3.10)$ & & 230 & $1.81(0.8-4.1)$ & 0.152 \\
\hline \multirow{2}{*}{ Collisson subtypes } & Exocrine-like vs. Classical & 245 & $1.13(0.74-1.70)$ & 0.131 & 230 & $1.75(0.98-3.12)$ & 0.057 \\
\hline & Quasi-mesenchymal vs. Classical & & $1.64(1.01-2.66)$ & & 230 & $1.12(0.63-2.01)$ & 0.698 \\
\hline Moffitt subtypes, “Tumor” & Classical vs. Basal-like & 245 & $0.53(0.37-0.77)$ & 0.001 & 230 & $0.55(0.31-1)$ & 0.050 \\
\hline Moffitt subtypes, "Stroma" & Normal vs. Activated & 240 & $0.46(0.3-0.69)$ & 0.0002 & 230 & $0.58(0.36-0.93)$ & 0.023 \\
\hline
\end{tabular}

ADEX: aberrantly differentiated endocrine, CI confidence interval, HR hazard ratio; $p$-value in bold: statistically significant. 
We looked at how the molecular classification could affect the survival in each of the two location groups. Notably, tumors from the "squamous" subtype from the Bailey's classification had a worse overall survival when the tumor was from the "B/T group" than from the "head group" (2-years OS: $0 \%$ versus $31 \%, p<0.0001$ ) (Figure 2 ). It was not possible to conclude for the immunological subtype because "B/ $\mathrm{T}$ " tumors included only one case. No difference in OS was observed with the other molecular subtypes according to the PDAC location.

A.

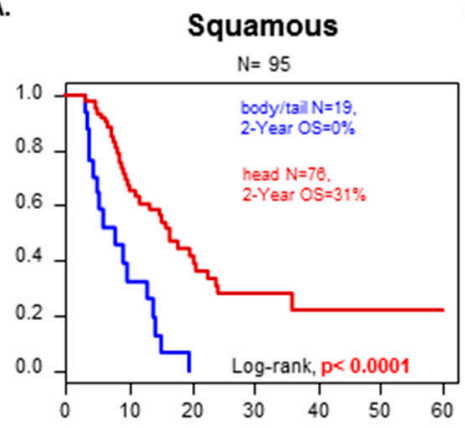

c.

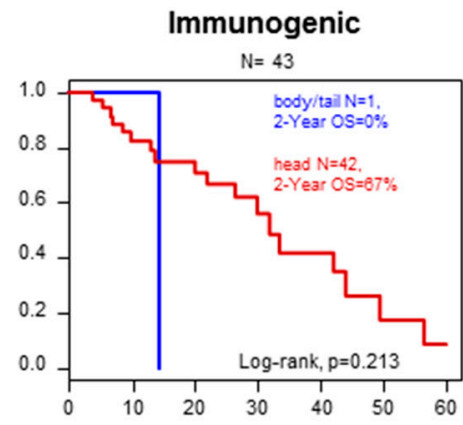

B.

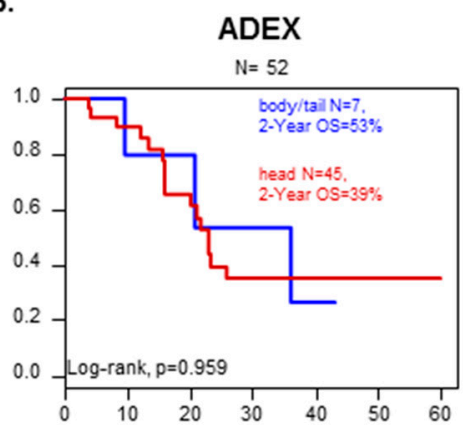

D.

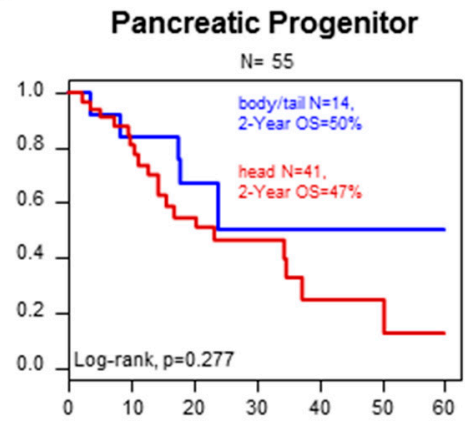

Figure 2. OS in the validation set according to Head or Body/Tail in the different Bailey's molecular subtypes. Kaplan-Meier OS curves according to PDAC location (Head vs. B/T) and the molecular subtypes defined by Bailey (A) Squamous, (B) ADEX, and (C) Immunogenic, and (D) Pancreatic progenitor. The $p$-values (log-rank test) for the comparison between the two classes within each molecular subtype are indicated.

\subsection{Head versus Body/Tail Gene Expression Signature}

To identify a gene expression signature (GES) of "head" versus "B/T" PDAC, we used the TCGA cohort as a learning set $(n=137)$ and the other sets pooled as a validation set $(n=112)$. A total of 334 genes were differentially expressed, including 278 (83\%) genes upregulated and 56 (17\%) genes downregulated in the "head group" compared to the "B/T group" (Supplementary Tables S2 and S3). A "location" classifier was then built from this gene list, and as expected, accurately classified the B/T samples according to their actual location (Fisher's exact test, $p<0.0001$ ). Importantly, its robustness as a classifier for B/T samples was confirmed in the independent validation set ( $p=0.0009$, Fisher's exact test) (Figure 3). 


\section{A. Learning set, GES 334 genes} moderated t-test, $p<5 \% \& q<25 \% \&|F C|>2 x$

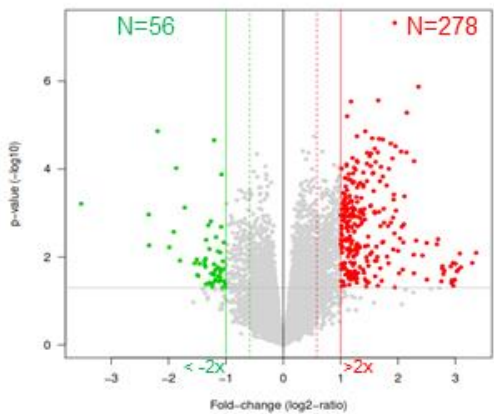

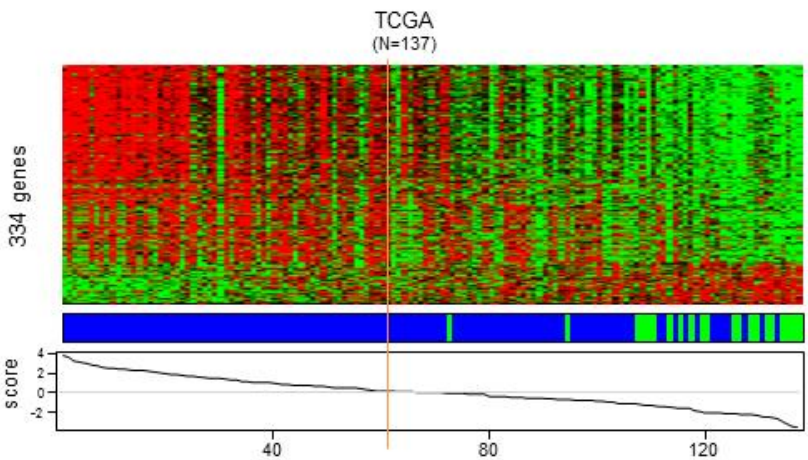

B. Validation sets

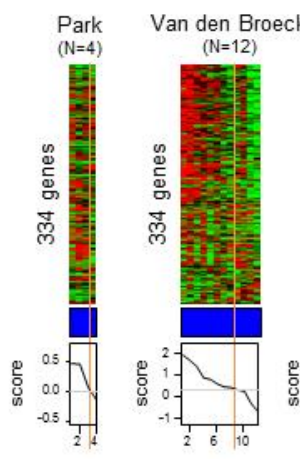

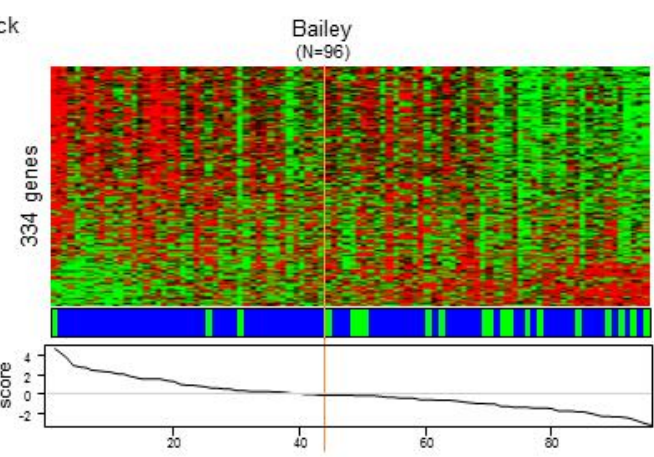

\section{Contingency analysis}

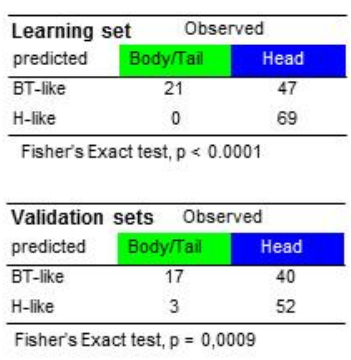

Figure 3. Establishment of the 334-gene expression signature between Head and $\mathrm{B} / \mathrm{T}$ tumors based on the learning and validation sets. (A) Volcano plot identifying 334 genes differentially expressed (GES) between Head and B/T tumors. This volcano plot was obtained using a moderated $t$-test, $p<5 \%$ \& $q<25 \%,|\mathrm{FC}|>2 \times$ between the Head and B/T tumors (left). The GES was used to classify the samples from the TCGA learning set (right). (B) Classification of the samples from each of the three validation sets using the 334 GES. (C) Contingency analyses of the classification in the learning and validation sets using the 334 GES identified from the learning set.

Regarding the 278 genes identified as upregulated in the "head group" (Supplementary Tables S2 and S3), two major pathways were noticeable. The first one was related to immune response activation $(p<0.0001)$, more specifically involving genes from inflammatory response $(p<0.0001)$, adaptive immune response $(p<0.0001)$, G-protein-coupled receptor signaling pathway $(p<0.0001)$, transmembrane receptor protein tyrosine kinase signaling $(p<0.0001)$, cell surface receptor signaling $(p<0.0001)$, and cellular defense response $(p<0.0001)$ pathways. Such activation of "Immune-related" pathways is coherent with above-mentioned enrichment of the immunogenic subtype in the "head group". The immune signature showed a strong immune infiltrate, more specifically involving upregulation of mRNA related to unconventional $\gamma \delta$-T cells $(p<0.0001)$, to the presence of a cytotoxic activity potentially at work and B-cells-related markers $(p<0.003)$. Finally, numerous cytokines, chemokines, and related receptors were secreted in "head" tumors and involved in immune cell recruitment and function $(p<0.0001)$.

The second major pathway upregulated in the "head" PDAC was related to pancreas function, notably triglyceride catabolic process $(p<0.0001)$, proteolysis $(p<0.0001)$, and digestion $(p<0.0001)$ process. These genes were related to pancreatic secretion $(p<0.0001)$, fat, protein and vitamin digestion and absorption $(p<0.0001)$, and glycolysis/gluconeogenesis/drug metabolism $(p<0.01)$.

Regarding the 56 genes identified as downregulated in the "head" compared to "B/T" PDAC (Supplementary Tables S2 and S3), associated pathways were involved in epidermis development and keratinocyte differentiation $(p<0.0001)$, more specifically to squamous tumors differentiation. Some 
of the downregulated genes were also related to the nervous system, notably dopaminergic synaptic transmission $(p<0.0001)$ and positive regulation of calcium ion import $(p<0.0001)$ that function as regulators of pancreas endocrine function, such as glucagon synthesis and positive regulation of cAMP biosynthetic process $(p<0.0001)$. Finally, several genes were related to tumor aggressiveness in "B/T" tumors, including genes involved in cellular proliferation, survival, and epithelial to mesenchymal transition. Some of these genes are associated components of the EGFR and/or ERBB and/or TP53 pathways. Other genes are involved in the regulation of cell proliferation and local invasion and the acquisition of drug resistance mechanisms and/or TGF- $\beta$-induced EMT.

\subsection{Correlation between "Head" vs. "Body/Tail" Locations and Immune and Stromal Signatures}

Because tissues are complex mixtures consisting of numerous noncancerous cell types, we searched for correlation between "head" vs. "body/tail" transcriptomic profiles and a variety of innate and adaptive immune cells, stromal cells, and many other cell types that are found in the tumor. For this, we used the XCELL [35] and the Gatza [39] gene expression signatures. In brief, the Gatza gene signature-based data aims at identifying enrichment for distinct oncogenic pathway activities; the XCELL gene signature-based data allow identifying immune and stromal cell types present within the tumor.

Those signatures showed correlations between the "head" transcriptional PDAC profile and the $\mathrm{T}$ and $\mathrm{B}$ immune infiltrates (CD8pos_Tcm, CD4pos_T.cells, naive_B.cells, B.cells, and Memory_B.cells) as well as some antigen-presenting cells (pDC, aDC, and B cells). Accordingly, the Microenvironment_Score $(p=0.0005)$ and Immune_Score $(p=0.0006)$ were significantly correlated with the "head" PDAC (Supplementary Table S4). By contrast, the "B/T" PDAC transcriptional profile was correlated with Keratinocytes ( $p=0.0007)$, Epithelial_cells $(p=0.0017)$, Smooth_muscle $(p=0.0123)$, and TGF $\beta(p=0.0299)$ gene signature-based profiles. As mentioned earlier, these signatures are suggestive of ongoing epithelial-to-mesenchymal transition (EMT) process. Altogether, these results confirmed the results of our "location" GES in term of differential pathways involved in the "head" versus " $\mathrm{B} / \mathrm{T}$ " PDAC.

\section{Discussion}

Currently, the AJCC TNM classification is the only prognostic factor used in clinical practice to assess survival of resected PDAC and guide treatments decision. However, recent data suggest that PDAC localized in the "head" might have a better survival than PDAC localized in the "B/ $\mathrm{T}$ " zone of the pancreas [20,27]. So far, this information is not taken into account when using the TNM classification. Still, the association between PDAC location and survival has been the subject of a longstanding debate, which is not completely settled yet. Here, we reported longer 2-year OS in "head" PDAC than in "B/T" PDAC. It has been hypothesized that "head" PDAC comes to clinical attention earlier than " $\mathrm{B} / \mathrm{T}$ " $\mathrm{PDC}$ due to early detectable symptoms, such as high carbohydrate antigen 19-9 (CA19-9) positivity or painless jaundice (caused by tumor obstruction of the bile ducts, which pass through the head of the pancreas). Thus, clinicians are prompt to perform abdominal imaging that will reveal the underlying tumor, and may thus deal with "earlier" stages of PDAC tumors. In contrast, tumors of the body and tail do not produce jaundice, and therefore most often come to clinical attention later, once weight loss and/or abdominal pain become apparent $[20,27,40]$. However, reciprocally, patients primarily diagnosed with pancreatic " $\mathrm{B} / \mathrm{T}$ " cancer are associated with much more pain, higher serum albumin level, higher carcinoembryonic antigen (CEA), and higher metastasis rate. Whatever the cause of detection, at diagnosis, " $\mathrm{B} / \mathrm{T}$ " $\mathrm{PDAC}$ corresponds to larger tumors that bear numerous molecular alterations, suggesting a later stage of disease evolution [20,41-48]. They are also more heterogeneous at the genomic level [49]. To avoid bias that can affect survival, we compared tumors with the same histological TNM classification. We confirmed that "B/T" PDAC has the worst 2-year OS for T3 and T4 N0 M0 tumors. This was not significant in the other groups, most certainly due to an inadequate number of samples in the analyzed groups despite our large cohort. 
In multivariate analysis, the $\mathrm{pN}$ status, the PDAC location and the Moffitt subtypes were the only three significant factors. The stratified hazard ratio (HR) for death was 0.46 ( $95 \%$ CI $0.27-0.79)$ in the head versus $\mathrm{B} / \mathrm{T}$ classes. We thus confirmed than PDAC localization is an independent prognostic factor when compared with the molecular subtype classifications. Of note, the location remained an independent prognostic factor in multivariate analysis when analysis was limited to the ductal tumors only (data not shown). We cannot exclude that this difference in survival might be inherent to other characteristics. We may however rule out the impact of the surgical procedure associated to the location (distal pancreatectomy versus pancreaticoduodenectomy). Indeed, when considering the survival of PDAC according to the molecular subtype classification (Figure 2B-D, $n=150$ cases), there was no significant difference in survival between the Head and the $\mathrm{B} / \mathrm{T}$ groups except for the Squamous subtype. If location and associated surgery only had an impact on survival, it would have been detectable for all molecular subtypes of PDAC, and not the Squamous subtype only. It is also possible that the molecular differences related to the head versus $\mathrm{B} / \mathrm{T}$ locations are not tackled by the molecular subtypes.

Overall, we were interested in defining the differential gene expression signature related to PDAC location. A recent study used pre-established "genes programs" issued from the PDAC classification of Bailey [33] to conduct a similar analysis [28]. It showed that the location in the " $B / T$ " was associated with the squamous subtype of PDAC, the molecular subtype with the worse clinical outcome. In addition to this finding, we reported a worst survival for squamous tumors localized in the $\mathrm{B} / \mathrm{T}$ as compared to the ones isolated from the "head" of the pancreas. This means that, independently from the squamous classification, the PDAC location has a prognostic impact. To go further with minimal induced bias (which can be introduced using pre-established gene programs), we did a global supervised analysis on a large learning dataset, then validated the obtained GES on an independent set. By doing so, we qualitatively identified the transcriptional difference between the two PDAC anatomic locations.

In line with the shorter survival of B/T tumors, our GES analysis between "head" and "B/T" PDAC revealed that $\mathrm{B} / \mathrm{T}$ tumors were probably more aggressive tumors, being highly proliferative and more prone to EMT. The higher proliferation rate could explain why " $\mathrm{B} / \mathrm{T}$ " tumors often display bigger size at diagnosis [28]. Tumor recurrence might also be related to this EMT-prone status [28]. Interestingly, these effects (proliferation and EMT status) seem to be amenable to EGFR and or ERBB2 regulation. TNS4, NTSR1, and PNCK are three genes involved in these pathways. Tensin-4 (or TNS4) functions as an oncogene and promotes cell proliferation and/or motility in numerous solid tumors, including pancreatic cancer [50], gastric cancer, hepatocellular carcinoma, and colorectal cancers. TNS4 is upregulated by EGF-induced ERK1/2 activity and KRAS, and as a retro-control loop, TNS4 regulates EGFR expression. Similarly, NTSR1, which belongs to the large superfamily of G-protein coupled receptors, was shown to promote tumor invasion by accelerating EMT [50]. NTSR1 could also affect cancer cells proliferation. High NTSR1 expression is associated with pancreatic ductal adenocarcinoma [51] and is a poor prognosis factor. Finally, PNCK, a member of the calcium/calmodulin-dependent protein kinase family of protein serine/threonine kinases, was shown to induce ligand-independent EGFR degradation. High expression of PNCK resulted in increased proliferation, clonal growth, cell cycle progression, and trastuzumab resistance in ERBB2-positive tumor cells [52-54]. Altogether, those three genes deserve more attention and might represent attractive therapeutic targets for " $\mathrm{B} / \mathrm{T}$ " $\mathrm{PDAC}$.

Because Langerhans islet concentration is much higher in the tail than in the head of the pancreas, theoretically, patients with pancreatic " $\mathrm{B} / \mathrm{T}$ " cancer are more prone to have islet dysfunction and subsequent diabetes. Data comparing the onset of pancreatic cancer-induced diabetes between "head" and " $\mathrm{B} / \mathrm{T}$ " PDAC are limited. Here, we have shown that the endocrine functions of the pancreas seem to be relatively more efficient in the "B/T" than in the "head" PDAC. Even in the presence of the growing tumor, the endocrine cells, notably insulin-secreting cells, seem to maintain their function. Insulin-positive endocrine cells seem to be "refractory" to malignant progression. This can explain why postoperative diabetes is higher in patients receiving distal $(\mathrm{B} / \mathrm{T})$ pancreatectomy than in those treated 
with pancreaticoduodenectomy $[8,55]$ and the delayed diagnosis of " $\mathrm{B} / \mathrm{T}$ " tumors in the absence of any detectable symptoms.

Another striking information related to our analysis was the absence of the immunological subtype in " $\mathrm{B} / \mathrm{T}$ " PDAC. This highlights a profound defect in the recruitment of leukocytes within "B/ $\mathrm{T}$ " PDAC and suggests that these tumors are probably noneligible for immune therapy-based treatment. The obvious defect in immune response was mainly noticeable due to the absence of CD8 $\gamma \delta-\mathrm{T}$ cells and $\mathrm{B}$ cells in " $\mathrm{B} / \mathrm{T}$ " PDAC. Because this transcriptomic analysis results from the whole tumor piece, we cannot know whether these cells were infiltrating or surrounding the tumor. These cells however overexpressed immune checkpoint inhibitory molecules, such as PD1, BTLA, TNFSF14 (LIGHT), and TIGIT, which are the sign of immune exhaustion or inhibition of tumor infiltrating lymphocytes. Gamma-delta T cells are part of the unconventional $\mathrm{T}$ cells involved in the initiation and propagation of immune responses. It is thus plausible that the reactivation of this cytotoxic immune infiltrate, achievable with the help of immune checkpoint inhibitors and/or potent analogues with more or less selective stimulatory activity for $\gamma \delta$-T cells (such as bromohydrin diphosphate (BrHPP), anti-KIR, phosphoantigen, or ligation of NKG2D, for example), can benefit to "head" PDAC. As for many solid tumors, boosting the immune system, especially with neoadjuvant therapeutic agents might be highly beneficial for "head" PDAC.

\section{Conclusions}

In conclusion, we have identified a robust GES associated with B/T PDAC samples. The nature of genes differentially expressed suggests that different therapeutic managements might be applied in clinics depending on the "head" or the " $\mathrm{B} / \mathrm{T}$ " location of tumor (Figure 4 ).

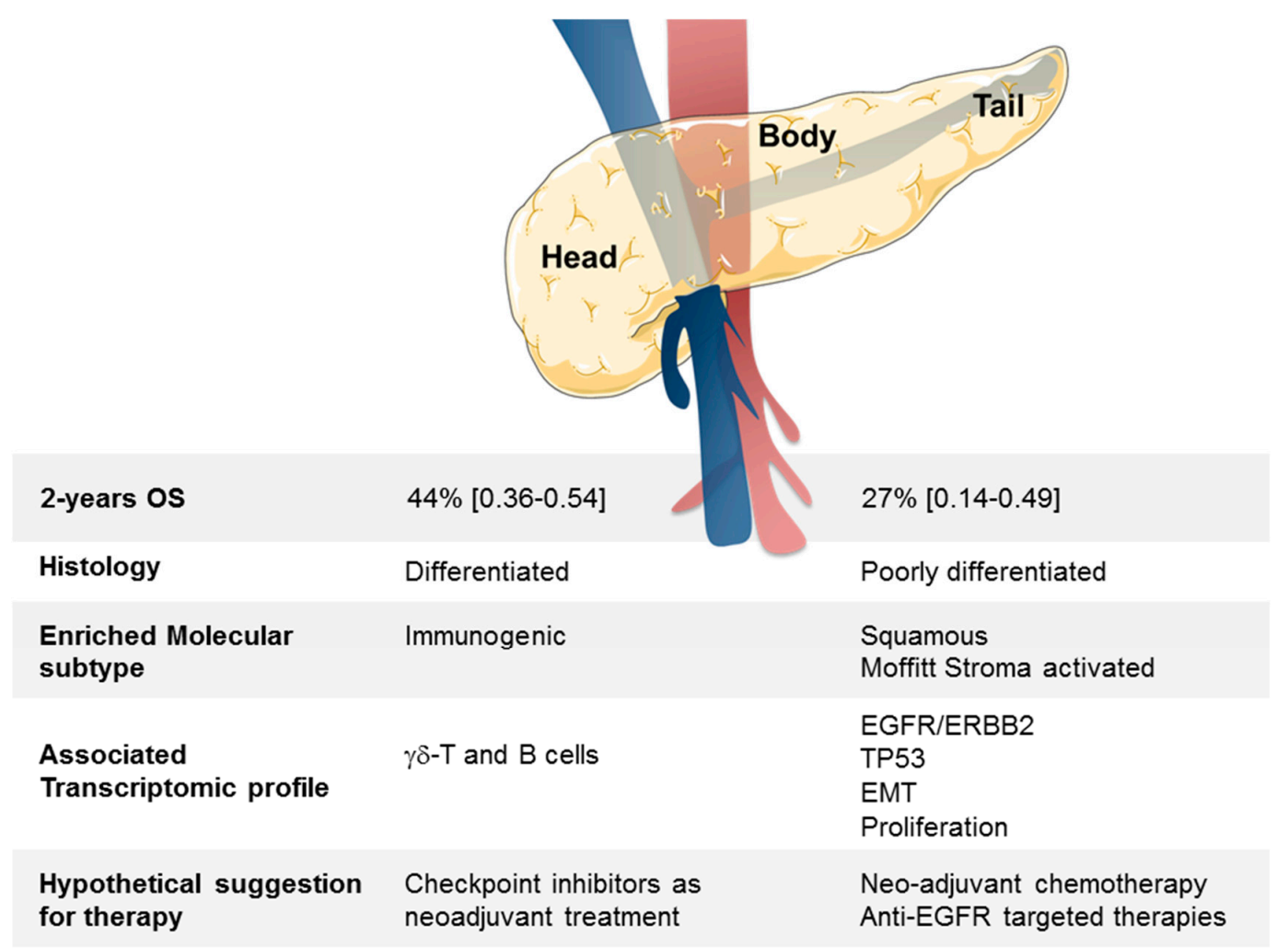

Figure 4. Summary of clinical, histologic, and molecular differences between head and B/T PDAC tumors. 
Supplementary Materials: The following are available online at http://www.mdpi.com/2072-6694/11/4/497/s1, Table S1: List of pancreatic cancer data sets included. Table S2: List of 334 genes differentially expressed between head and B/T location of PDAC. Table S3: Ontology analysis of the 334 genes differentially expressed between the head and B/T location of PDAC. Table S4: Enrichment for cell types and or oncogenic pathway activities in "head" vs. "body/tail" samples. Figure S1. Principal component analysis performed before and after normalization of the 4 data sets.

Author Contributions: Conceptualization, D.J.B. and F.B.; Formal analysis, P.F.; Methodology, P.F.; Supervision, D.B. and E.M.; Validation, D.J.B.; Writing—original draft, D.J.B.; Writing—review \& editing, D.J.B., F.B., D.B. and E.M.

Funding: This research was funded by Inserm/Institut Paoli-Calmettes Grant SIRIC INCa-DGOS-Inserm 6038.

Conflicts of Interest: The authors declare no conflicts of interest.

\section{References}

1. Jemal, A.; Bray, F.; Center, M.M.; Ferlay, J.; Ward, E.; Forman, D. Global cancer statistics. CA Cancer J. Clin. 2011, 61, 69-90. [CrossRef] [PubMed]

2. Rahib, L.; Smith, B.D.; Aizenberg, R.; Rosenzweig, A.B.; Fleshman, J.M.; Matrisian, L.M. Projecting cancer incidence and deaths to 2030: The unexpected burden of thyroid, liver, and pancreas cancers in the United States. Cancer Res. 2014, 74, 2913-2921. [CrossRef]

3. Ducreux, M.; Cuhna, A.S.; Caramella, C.; Hollebecque, A.; Burtin, P.; Goere, D.; Seufferlein, T.; Haustermans, K.; Van Laethem, J.L.; Conroy, T.; et al. Cancer of the pancreas: ESMO Clinical Practice Guidelines for diagnosis, treatment and follow-up. Ann. Oncol. 2015, 26, v56-v68. [CrossRef] [PubMed]

4. Neoptolemos, J.P.; Palmer, D.H.; Ghaneh, P.; Psarelli, E.E.; Valle, J.W.; Halloran, C.M.; Faluyi, O.; O’Reilly, D.A.; Cunningham, D.; Wadsley, J.; et al. Comparison of adjuvant gemcitabine and capecitabine with gemcitabine monotherapy in patients with resected pancreatic cancer (ESPAC-4): A multicentre, open-label, randomised, phase 3 trial. Lancet 2017, 389, 1011-1024. [CrossRef]

5. Nowak, N.J.; Gaile, D.; Conroy, J.M.; McQuaid, D.; Cowell, J.; Carter, R.; Goggins, M.G.; Hruban, R.H.; Maitra, A. Genome-wide aberrations in pancreatic adenocarcinoma. Cancer Genet. Cytogenet. 2005, 161, 36-50. [CrossRef] [PubMed]

6. Buchler, M.; Friess, H.; Schultheiss, K.H.; Gebhardt, C.; Kubel, R.; Muhrer, K.H.; Winkelmann, M.; Wagener, T.; Klapdor, R.; Kaul, M.; et al. A randomized controlled trial of adjuvant immunotherapy (murine monoclonal antibody 494/32) in resectable pancreatic cancer. Cancer 1991, 68, 1507-1512. [CrossRef]

7. Friess, H.; Buchler, M.; Kruger, M.; Beger, H.G. Treatment of duct carcinoma of the pancreas with the LH-RH analogue buserelin. Pancreas 1992, 7, 516-521.

8. Gidekel Friedlander, S.Y.; Chu, G.C.; Snyder, E.L.; Girnius, N.; Dibelius, G.; Crowley, D.; Vasile, E.; DePinho, R.A.; Jacks, T. Context-dependent transformation of adult pancreatic cells by oncogenic K-Ras. Cancer Cell 2009, 16, 379-389. [CrossRef] [PubMed]

9. Hori, M.; Kitahashi, T.; Imai, T.; Ishigamori, R.; Takasu, S.; Mutoh, M.; Sugimura, T.; Wakabayashi, K.; Takahashi, M. Enhancement of carcinogenesis and fatty infiltration in the pancreas in $\mathrm{N}$-nitrosobis(2-oxopropyl)amine-treated hamsters by high-fat diet. Pancreas 2011, 40, 1234-1240. [CrossRef]

10. Kawamoto, S.; Siegelman, S.S.; Bluemke, D.A.; Hruban, R.H.; Fishman, E.K. Focal fatty infiltration in the head of the pancreas: Evaluation with multidetector computed tomography with multiplanar reformation imaging. J. Comput. Assist. Tomogr. 2009, 33, 90-95. [CrossRef] [PubMed]

11. Pitman, M.B.; Lewandrowski, K.; Shen, J.; Sahani, D.; Brugge, W.; Fernandez-del Castillo, C. Pancreatic cysts: Preoperative diagnosis and clinical management. Cancer Cytopathol. 2010, 118, 1-13. [CrossRef]

12. Khashab, M.A.; Shin, E.J.; Amateau, S.; Canto, M.I.; Hruban, R.H.; Fishman, E.K.; Cameron, J.L.; Edil, B.H.; Wolfgang, C.L.; Schulick, R.D.; et al. Tumor size and location correlate with behavior of pancreatic serous cystic neoplasms. Am. J. Gastroenterol. 2011, 106, 1521-1526. [CrossRef]

13. Park, J.; Lee, K.T.; Jang, T.H.; Seo, Y.W.; Lee, K.H.; Lee, J.K.; Jang, K.T.; Heo, J.S.; Choi, S.H.; Choi, D.W.; et al. Risk factors associated with the postoperative recurrence of intraductal papillary mucinous neoplasms of the pancreas. Pancreas 2011, 40, 46-51. [CrossRef]

14. Oh, T.G.; Chung, M.J.; Park, J.Y.; Bang, S.M.; Park, S.W.; Chung, J.B.; Song, S.Y. Prognostic factors and characteristics of pancreatic neuroendocrine tumors: Single center experience. Yonsei Med. J. 2012, 53, 944-951. [CrossRef] [PubMed] 
15. Ruess, D.A.; Makowiec, F.; Chikhladze, S.; Sick, O.; Riediger, H.; Hopt, U.T.; Wittel, U.A. The prognostic influence of intrapancreatic tumor location on survival after resection of pancreatic ductal adenocarcinoma. BMC Surg. 2015, 15, 123. [CrossRef]

16. Van Erning, F.N.; Mackay, T.M.; van der Geest, L.G.M.; Groot Koerkamp, B.; van Laarhoven, H.W.M.; Bonsing, B.A.; Wilmink, J.W.; van Santvoort, H.C.; de Vos-Geelen, J.; van Eijck, C.H.J.; et al. Association of the location of pancreatic ductal adenocarcinoma (head, body, tail) with tumor stage, treatment, and survival: A population-based analysis. Acta Oncol. 2018, 1-8. [CrossRef] [PubMed]

17. Chang, D.T.; Schellenberg, D.; Shen, J.; Kim, J.; Goodman, K.A.; Fisher, G.A.; Ford, J.M.; Desser, T.; Quon, A.; Koong, A.C. Stereotactic radiotherapy for unresectable adenocarcinoma of the pancreas. Cancer 2009, 115, 665-672. [CrossRef]

18. Marechal, R.; Demols, A.; Gay, F.; De Maertelaere, V.; Arvanitaki, M.; Hendlisz, A.; Van Laethem, J.L. Prognostic factors and prognostic index for chemonaive and gemcitabine-refractory patients with advanced pancreatic cancer. Oncology 2007, 73, 41-51. [PubMed]

19. Tanaka, T.; Ikeda, M.; Okusaka, T.; Ueno, H.; Morizane, C.; Hagihara, A.; Iwasa, S.; Kojima, Y. Prognostic factors in japanese patients with advanced pancreatic cancer treated with single-agent gemcitabine as first-line therapy. Jpn. J. Clin. Oncol. 2008, 38, 755-761. [CrossRef]

20. Lau, M.K.; Davila, J.A.; Shaib, Y.H. Incidence and survival of pancreatic head and body and tail cancers: A population-based study in the United States. Pancreas 2010, 39, 458-462. [CrossRef]

21. Sener, S.F.; Fremgen, A.; Menck, H.R.; Winchester, D.P. Pancreatic cancer: A report of treatment and survival trends for 100,313 patients diagnosed from 1985-1995, using the National Cancer Database. J. Am. Coll. Surg. 1999, 189, 1-7. [CrossRef]

22. Sohn, T.A.; Yeo, C.J.; Cameron, J.L.; Koniaris, L.; Kaushal, S.; Abrams, R.A.; Sauter, P.K.; Coleman, J.; Hruban, R.H.; Lillemoe, K.D. Resected adenocarcinoma of the pancreas-616 patients: Results, outcomes, and prognostic indicators. J. Gastrointest. Surg. 2000, 4, 567-579. [CrossRef]

23. Toomey, P.; Hernandez, J.; Golkar, F.; Ross, S.; Luberice, K.; Rosemurgy, A. Pancreatic adenocarcinoma: Complete tumor extirpation improves survival benefit despite larger tumors for patients who undergo distal pancreatectomy and splenectomy. J. Gastrointest. Surg. 2012, 16, 376-381. [CrossRef]

24. Dalton, R.R.; Sarr, M.G.; van Heerden, J.A.; Colby, T.V. Carcinoma of the body and tail of the pancreas: Is curative resection justified? Surgery 1992, 111, 489-494. [PubMed]

25. Brennan, M.F.; Moccia, R.D.; Klimstra, D. Management of adenocarcinoma of the body and tail of the pancreas. Ann. Surg. 1996, 223, 506-511; discussion 511-512. [CrossRef] [PubMed]

26. Sperti, C.; Pasquali, C.; Piccoli, A.; Pedrazzoli, S. Survival after resection for ductal adenocarcinoma of the pancreas. Br. J. Surg. 1996, 83, 625-631. [CrossRef] [PubMed]

27. Artinyan, A.; Soriano, P.A.; Prendergast, C.; Low, T.; Ellenhorn, J.D.; Kim, J. The anatomic location of pancreatic cancer is a prognostic factor for survival. HPB (Oxford) 2008, 10, 371-376. [CrossRef] [PubMed]

28. Dreyer, S.B.; Jamieson, N.B.; Upstill-Goddard, R.; Bailey, P.J.; McKay, C.J.; Biankin, A.V.; Chang, D.K. Defining the molecular pathology of pancreatic body and tail adenocarcinoma. Br. J. Surg. 2018, 105, e183-e191. [CrossRef] [PubMed]

29. Irizarry, R.A.; Bolstad, B.M.; Collin, F.; Cope, L.M.; Hobbs, B.; Speed, T.P. Summaries of Affymetrix GeneChip probe level data. Nucleic Acids Res. 2003, 31, e15. [CrossRef] [PubMed]

30. Eisen, M.B.; Spellman, P.T.; Brown, P.O.; Botstein, D. Cluster analysis and display of genome-wide expression patterns. Proc. Natl. Acad. Sci. USA 1998, 95, 14863-14868. [CrossRef] [PubMed]

31. Collisson, E.A.; Sadanandam, A.; Olson, P.; Gibb, W.J.; Truitt, M.; Gu, S.; Cooc, J.; Weinkle, J.; Kim, G.E.; Jakkula, L.; et al. Subtypes of pancreatic ductal adenocarcinoma and their differing responses to therapy. Nat. Med. 2011, 17, 500-503. [CrossRef] [PubMed]

32. Moffitt, R.A.; Marayati, R.; Flate, E.L.; Volmar, K.E.; Loeza, S.G.; Hoadley, K.A.; Rashid, N.U.; Williams, L.A.; Eaton, S.C.; Chung, A.H.; et al. Virtual microdissection identifies distinct tumor- and stroma-specific subtypes of pancreatic ductal adenocarcinoma. Nat. Genet. 2015, 47, 1168-1178. [CrossRef]

33. Bailey, P.; Chang, D.K.; Nones, K.; Johns, A.L.; Patch, A.M.; Gingras, M.C.; Miller, D.K.; Christ, A.N.; Bruxner, T.J.; Quinn, M.C.; et al. Genomic analyses identify molecular subtypes of pancreatic cancer. Nature 2016, 531, 47-52. [CrossRef] [PubMed] 
34. Gatza, M.L.; Lucas, J.E.; Barry, W.T.; Kim, J.W.; Wang, Q.; Crawford, M.D.; Datto, M.B.; Kelley, M.; Mathey-Prevot, B.; Potti, A.; et al. A pathway-based classification of human breast cancer. Proc. Natl. Acad. Sci. USA 2010, 107, 6994-6999. [CrossRef] [PubMed]

35. Aran, D.; Hu, Z.; Butte, A.J. xCell: Digitally portraying the tissue cellular heterogeneity landscape. Genome Biol. 2017, 18, 220. [CrossRef]

36. Smyth, G.K. Linear models and empirical bayes methods for assessing differential expression in microarray experiments. Stat. Appl. Genet. Mol. Biol. 2004, 3, 1-25. [CrossRef] [PubMed]

37. Da Huang, W.; Sherman, B.T.; Lempicki, R.A. Systematic and integrative analysis of large gene lists using DAVID bioinformatics resources. Nat. Protoc. 2009, 4, 44-57. [CrossRef] [PubMed]

38. McShane, L.M.; Altman, D.G.; Sauerbrei, W.; Taube, S.E.; Gion, M.; Clark, G.M. REporting recommendations for tumour MARKer prognostic studies (REMARK). Br. J. Cancer 2005, 93, 387-391. [CrossRef] [PubMed]

39. Gatza, M.L.; Kung, H.N.; Blackwell, K.L.; Dewhirst, M.W.; Marks, J.R.; Chi, J.T. Analysis of tumor environmental response and oncogenic pathway activation identifies distinct basal and luminal features in HER2-related breast tumor subtypes. Breast Cancer Res. 2011, 13, R62. [CrossRef]

40. Watanabe, I.; Sasaki, S.; Konishi, M.; Nakagohri, T.; Inoue, K.; Oda, T.; Kinoshita, T. Onset symptoms and tumor locations as prognostic factors of pancreatic cancer. Pancreas 2004, 28, 160-165. [CrossRef] [PubMed]

41. Bilimoria, K.Y.; Bentrem, D.J.; Ko, C.Y.; Stewart, A.K.; Winchester, D.P.; Talamonti, M.S. National failure to operate on early stage pancreatic cancer. Ann. Surg. 2007, 246, 173-180. [CrossRef]

42. Matsuno, S.; Egawa, S.; Fukuyama, S.; Motoi, F.; Sunamura, M.; Isaji, S.; Imaizumi, T.; Okada, S.; Kato, H.; Suda, K.; et al. Pancreatic Cancer Registry in Japan: 20 years of experience. Pancreas 2004, 28, 219-230. [CrossRef]

43. Van Oost, F.J.; Luiten, E.J.; van de Poll-Franse, L.V.; Coebergh, J.W.; van den Eijnden-van Raaij, A.J. Outcome of surgical treatment of pancreatic, peri-ampullary and ampullary cancer diagnosed in the south of The Netherlands: A cancer registry based study. Eur. J. Surg. Oncol. 2006, 32, 548-552. [CrossRef] [PubMed]

44. Wu, T.C.; Shao, Y.F.; Shan, Y.; Wu, J.X.; Zhao, P. Surgical effect of malignant tumor of body and tail of the pancreas: Compare with pancreatic head cancer. Zhonghua Wai Ke Za Zhi 2007, 45, 30-33. [PubMed]

45. Notta, F.; Chan-Seng-Yue, M.; Lemire, M.; Li, Y.; Wilson, G.W.; Connor, A.A.; Denroche, R.E.; Liang, S.B.; Brown, A.M.; Kim, J.C.; et al. A renewed model of pancreatic cancer evolution based on genomic rearrangement patterns. Nature 2016, 538, 378-382. [CrossRef] [PubMed]

46. Jones, S.; Zhang, X.; Parsons, D.W.; Lin, J.C.; Leary, R.J.; Angenendt, P.; Mankoo, P.; Carter, H.; Kamiyama, H.; Jimeno, A.; et al. Core signaling pathways in human pancreatic cancers revealed by global genomic analyses. Science 2008, 321, 1801-1806. [CrossRef] [PubMed]

47. Talbott, V.A.; Yeo, C.J.; Brody, J.R.; Witkiewicz, A.K. Molecular profiling of synchronous and metachronous cancers of the pancreas reveal molecular mimicry between samples from the same patient. J. Surg. Res. 2012, 176, 154-158. [CrossRef]

48. Eyigor, C.; Karaca, B.; Kuzeyli-Yildirim, Y.; Uslu, R.; Uyar, M.; Coker, A. Does the tumor localization in advanced pancreatic cancer have an influence on the management of symptoms and pain? J. Balk. Union Oncol. 2010, 15, 543-546.

49. Jamal-Hanjani, M.; Wilson, G.A.; McGranahan, N.; Birkbak, N.J.; Watkins, T.B.K.; Veeriah, S.; Shafi, S.; Johnson, D.H.; Mitter, R.; Rosenthal, R.; et al. Tracking the Evolution of Non-Small-Cell Lung Cancer. N. Engl. J. Med. 2017, 376, 2109-2121. [CrossRef]

50. Al-Ghamdi, S.; Cachat, J.; Albasri, A.; Ahmed, M.; Jackson, D.; Zaitoun, A.; Guppy, N.; Otto, W.R.; Alison, M.R.; Kindle, K.B.; et al. C-terminal tensin-like gene functions as an oncogene and promotes cell motility in pancreatic cancer. Pancreas 2013, 42, 135-140. [CrossRef]

51. Yin, X.; Wang, M.; Wang, H.; Deng, H.; He, T.; Tan, Y.; Zhu, Z.; Wu, Z.; Hu, S.; Li, Z. Evaluation of neurotensin receptor 1 as a potential imaging target in pancreatic ductal adenocarcinoma. Amino Acids 2017, 49, 1325-1335. [CrossRef] [PubMed]

52. Deb, T.B.; Zuo, A.H.; Barndt, R.J.; Sengupta, S.; Jankovic, R.; Johnson, M.D. Pnck overexpression in HER-2 gene-amplified breast cancer causes Trastuzumab resistance through a paradoxical PTEN-mediated process. Breast Cancer Res. Treat. 2015, 150, 347-361. [CrossRef] [PubMed]

53. Deb, T.B.; Zuo, A.H.; Wang, Y.; Barndt, R.J.; Cheema, A.K.; Sengupta, S.; Coticchia, C.M.; Johnson, M.D. Pnck induces ligand-independent EGFR degradation by probable perturbation of the Hsp90 chaperone complex. Am. J. Physiol. Cell Physiol. 2011, 300, C1139-C1154. [CrossRef] 
54. Deb, T.B.; Coticchia, C.M.; Barndt, R.; Zuo, H.; Dickson, R.B.; Johnson, M.D. Pregnancy-upregulated nonubiquitous calmodulin kinase induces ligand-independent EGFR degradation. Am. J. Physiol. Cell Physiol. 2008, 295, C365-C377. [CrossRef] [PubMed]

55. Malka, D.; Hammel, P.; Sauvanet, A.; Rufat, P.; O’Toole, D.; Bardet, P.; Belghiti, J.; Bernades, P.; Ruszniewski, P.; Levy, P. Risk factors for diabetes mellitus in chronic pancreatitis. Gastroenterology 2000, 119, 1324-1332. [CrossRef] [PubMed]

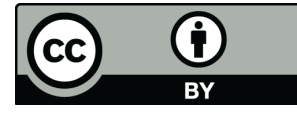

(C) 2019 by the authors. Licensee MDPI, Basel, Switzerland. This article is an open access article distributed under the terms and conditions of the Creative Commons Attribution (CC BY) license (http://creativecommons.org/licenses/by/4.0/). 